\title{
Subsequent Pregnancy Outcomes After Open Maternal-Fetal Surgery for Myelomeningocele
}

\author{
William H. Goodnight ${ }^{1}$, Ozan Bahtiyar ${ }^{2}$, Kelly A. Bennett ${ }^{3}$, Stephen P. Emery ${ }^{4}$, JB \\ Lillegard $^{5}$, Allan Fisher ${ }^{6}$, Ruth Goldstein ${ }^{7}$, Jillian Jatres ${ }^{8}$, Foong-Yen Lim ${ }^{9}$, Laurence \\ McCullough $^{10}$, Ueli Moehrlen ${ }^{11}$, Julie S. Moldenhauer ${ }^{8}$, Anita J. Moon-Grady ${ }^{12}$, Rodrigo \\ Ruano $^{13}$, Daniel W. Skupski ${ }^{14}$, Elizabeth Thom ${ }^{15}$, Marjorie C. Treadwell ${ }^{16}$, KuoJen Tsao ${ }^{17}$,

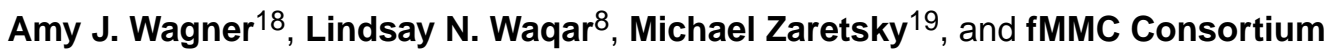 \\ sponsored by NAFTNet
}

\begin{abstract}
${ }^{1}$ University of North Carolina at Chapel Hill, Chapel Hill, NC, ${ }^{2}$ Yale University, New Haven, CT, ${ }^{3}$ Vanderbilt University, Nashville, TN, ${ }^{4}$ University of Pittsburgh, Pittsburgh, PA, ${ }^{5}$ Children's Hospital of Minnesota, Midwest Fetal Care Center, ${ }^{6}$ St Louis University, St Louis, MO, ${ }^{7}$ University of California San Francisco, San Francisco, CA, ${ }^{8}$ Children's Hospital of Philadelphia, Philadelphia, PA, ${ }^{9} \mathrm{Cincinnati}$ Children's Hospital, Cincinnati, OH, ${ }^{10}$ Baylor College of Medicine, Houston, TX, ${ }^{11}$ Children's Hospital Zurich, Zurich, Switzerland, ${ }^{12}$ University of California San Francisco, San Francisco, CA, ${ }^{13}$ Mayo Clinic, Rochester, MN, ${ }^{14}$ Cornell University, New York, NY, ${ }^{15}$ George Washington University, Bethesda, MD, ${ }^{16}$ University of Michigan, Ann Arbor, MI, ${ }^{17}$ University of Texas Health Center, Houston, TX, ${ }^{18}$ Children's Hospital of Wisconsin Fetal Concerns Center, Milwaukee, WI, ${ }^{19}$ Colorado Fetal Care Center, Denver, CO.
\end{abstract}

\section{Abstract}

Background: Open maternal-fetal surgery (OMFS) for fetal myelomeningocele (fMMC) results in reduction in neonatal morbidity related to spina bifida but may be associated with fetal, neonatal, and maternal complications in subsequent pregnancies.

Objective: The objective of this study was to ascertain obstetric risk in subsequent pregnancies after OMFS for fMMC closure.

Study Design: An international multicenter prospective observational registry created to track and report maternal, obstetric, fetal/neonatal and subsequent pregnancy outcomes following OMFS for fMMC, was evaluated for subsequent pregnancy outcome variables. Institutional Review Board approval was obtained for the registry.

Results: From 693 cases of OMFS for fMMC closure entered into the registry, 77 subsequent pregnancies in 60 women were identified. The overall live birth rate was $96.2 \%$ with 52

Corresponding author: William Goodnight, MD, MSCR, Department of Obstetrics and Gynecology, University of North Carolina School of Medicine, 3010 Old Clinic Building, CB \# 7516, Chapel Hill, NC 27599, william_goodnight@ med.unc.edu, 919-966-1601. Publisher's Disclaimer: This is a PDF file of an unedited manuscript that has been accepted for publication. As a service to our customers we are providing this early version of the manuscript. The manuscript will undergo copyediting, typesetting, and review of the resulting proof before it is published in its final citable form. Please note that during the production process errors may be discovered which could affect the content, and all legal disclaimers that apply to the journal pertain. 
pregnancies delivering beyond 20 weeks gestational age and median gestational age at delivery of 37 (36.3-37.1) weeks. The uterine rupture rate was 9.6\% ( $\mathrm{n}=5)$, resulting in 2 fetal deaths.

Maternal transfusion was required in 4 patients (7.7\%).

Conclusion: The risk of uterine rupture or dehiscence in subsequent pregnancies with associated fetal morbidity after OMFS is significant, but similar to that reported for subsequent pregnancies after classical cesarean deliveries. Future pregnancy considerations should be included in initial counseling for women contemplating OMFS.

\section{Keywords}

cesarean delivery; fetal myelomeningocele; fetal myelomeningocele repair; fetal surgery; fMMC; open maternal fetal surgery; myelomeningocele; spina bifida; uterine rupture

\section{Introduction}

The Management of Myelomeningocele Study (MOMS) demonstrated that open maternalfetal surgery (OMFS) for fetal myelomeningocele (fMMC) closure results in a significant reduction in morbidity associated with spina bifida, including reduction in the need for ventriculoperitoneal shunting in the first year of life, reversal of hindbrain herniation and improving motor function and neurodevelopmental outcomes at 30 months of age [1]. OMFS for fMMC is performed via a hysterotomy in the contractile portion of the uterus, similar to that created during a classical cesarean delivery.

Limited information exists regarding the influence of OMFS on subsequent pregnancy outcomes. As a classical hysterotomy is associated with a uterine rupture risk of 4-9\% which can be associated with increased maternal and neonatal morbidity [2,3], there is potential for similar or greater risk in pregnancy following OMFS. Wilson et al described the outcomes of 36 subsequent pregnancies that progressed beyond 20 weeks gestational age in women who had prior OMFS from 1996-2007 [4]. Fetal indications for OMFS in this group included fMMC, fetal lung malformation, sacrococcygeal teratoma, and congenital diaphragmatic hernia. In this cohort, uterine rupture or dehiscence in a subsequent pregnancy occurred in $14 \%$ and $14 \%$, respectively, with one uterine rupture resulting in cesarean hysterectomy and neonatal death due to prematurity. Zamora et al reported one subsequent pregnancy in a cohort of 7 patients undergoing OMFS, resulting in a uterine rupture and delivery at 36 weeks gestational age with surviving mother and neonate [5]. Of 16 women reporting subsequent pregnancies after OMFS in the MOMS trial, the uterine dehiscence rate was $12.5 \%$ in this limited series [6] without any reported uterine ruptures. Thus, limited data suggests a significant risk of uterine incision complications in pregnancies subsequent to OMFS, however the majority of the current reported literature includes indications for open maternal fetal surgery other than fMMC that could have dissimilar hysterotomy characteristics. Additionally, given the increase in number of OMFS using the MOMS criteria for fMMC closure and the potential for future fertility, precision of the estimate for reproductive outcomes beyond the current literature in the MMC population is needed. 
The Fetal Myelomeningocele Consortium sponsored by the North American Fetal Therapy Network (NAFTNet) was established in 2012 with the goal to provide a network for institutions performing fMMC closure and establish a prospective registry of patients undergoing fMMC closure after the MOMS trial. The goal of the registry is to systematically collect and analyze data related to maternal-fetal surgery for fMMC closure, to ensure continued patient outcomes comparable to the MOMS trial, and provide an opportunity for additional scientific investigation. The fMMC Consortium now consists of more than 25 collaborative institutions from around the world who perform fMMC closure.

The specific aims of this study are to describe the maternal, fetal and neonatal outcomes in subsequent pregnancies following a pregnancy that underwent OMFS for fMMC closure, using data from the fMMC Consortium registry.

\section{Materials and Methods:}

A prospective observational registry was created by the Fetal Myelomeningocele Consortium, sponsored by the North American Fetal Therapy Network. NAFTNet and the fMMC Consortium are supported through funding by the Eunice Kennedy Shriver National Institute of Child Health and Human Development (5R131HD059293-05), with NAFTNet support provided to create the registry. Currently, data entry and data analysis from this registry is dependent upon individual site resources. The registry was created to track maternal and neonatal outcomes following maternal-fetal surgery for fMMC closure after completion of the MOMS trial, as well as collect and analyze data on subsequent pregnancy outcomes. Individual institutions were invited to enroll patients being evaluated for fMMC closure. Any institution offering maternal-fetal surgery for fMMC closure is eligible to participate. Registry IRB was obtained by the Children's Hospital of Philadelphia as the coordinating center, and individual centers obtained IRB approval for prospective observation of consenting patients. Health Insurance Portability and Accountability Act authorization is obtained with the informed consent process. Retrospective data was obtained from prenatal and delivery records of the mother and neonatal records of the infant. Data collected from subsequent pregnancies is obtained by patient questionnaires performed by study personnel with confirmation of outcome based on review of medical records as available. Subsequent pregnancy outcome data include fetal number, gestational age at delivery, pregnancy outcome (live birth, pregnancy loss), uterine incision status at delivery, route of delivery and description of neonatal or maternal complications, including transfusion, hysterectomy, and maternal death.

Study data are collected and managed using REDCap electronic data capture tools hosted at The Children's Hospital of Philadelphia for the fMMC Consortium. REDCap (Research Electronic Data Capture) is a secure, web-based application designed to support data capture for research studies, providing an intuitive interface for validated data entry, audit trails for tracking data manipulation and export procedures, automated export procedures for seamless data downloads to common statistical packages, and procedures for importing data from external sources [7]. De-identified data are provided to the investigator for specific study questions using the registry data. The investigators are blinded to individual clinical site as well as patient identifying information. The data used for this analysis include patients who 
began evaluation for $\mathrm{AMMC}$ on or after 12/15/2010. The data for this analysis was last accessed 1/31/2019. IRB approval was obtained for the registry at the Children's Hospital of Philadelphia (IRB\# 11-008262, 9/20/2013), the coordinating center, and local IRB approved by the University of North Carolina at Chapel Hill (IRB 13-3452). Each participating center completes local IRB approval prior to receiving access to the registry. Pregnancy outcomes and demographic data were reported using descriptive statistics ( $\mathrm{N}(\%)$, median (interquartile range)). Comparison among groups was described using $\chi^{2}$ or Fisher exact for categorical data or Student's T test or Wilcoxon rank sum as appropriate. Statistical analysis was performed using SPSS (IBM SPSS Statistics 25, 2017). The method of OMFS and overall clinical care is determined by the operating clinical team and not dictated by the fMMC registry. Members of the fMMC Consortium report largely following the same inclusion criteria and surgical methods used in the MOMS trial[1, 8]. Hysterotomy incisions for OMFS are typically either vertical or transverse incisions in the contractile portion of the uterus, with position opposite the placental location. The uterine entry technique used in most North American centers performing fMMC closure includes developing a 1-2 cm uterine window with cautery or sharp dissection after two stay sutures are placed. The hysterotomy is then extended to create a 4-6 cm incision using a uterine stapling device with absorbable glycolide/lactide copolymer staples (Premium Poly CS, Coviden ${ }^{\mathrm{TM}}$, Medtronic) that results in a hemostatic uterine incision incorporating uterine serosa, myometrium and the chorionic and amniotic membranes. The hysterotomy is typically closed with two layers of $0.35 \mathrm{~mm}$ diameter delayed absorbable suture. Delivery is then performed at 37 weeks EGA, unless indications arise prior to that time for preterm delivery, typically through a separate low transverse uterine incision.

In this data, inter-delivery interval is defined as the interval in years from delivery of the OMFS pregnancy to delivery of the subsequent pregnancy. At the time of delivery in the OMFS pregnancy as well as the subsequent pregnancy, the fetal surgery hysterotomy site was described as intact, thinned, dehiscence (defined as disruption of the uterine mucosa with intact serosa), or rupture (defined as disruption of the uterine muscle and serosa). At the time of delivery of the subsequent pregnancy, the status of the hysterotomy incision was defined as 'thin or dehiscence' (yes/no). The prevalence of uterine rupture and live birth were calculated among pregnancies that progressed beyond 20 weeks EGA [9]. The registry does not currently contain detailed neonatal outcomes from subsequent pregnancies. The patients enrolled in this registry do not include MOMS trial patients and this is the first publication for this cohort of patients.

\section{Results:}

There are 16 institutions participating in the fMMC registry, of which 12 have actively entered patient data. In this registry, 1166 patients are included who have undergone evaluation and 693 (59.4\%) women ultimately underwent OMFS for fMMC closure. Among those that completed a pregnancy complicated by OMFS, 60 women reported 77 subsequent pregnancies. The demographic characteristics of this group is described in table 1. Forty-five women (75.0\%) reported one subsequent pregnancy, 13 women (21.7\%) reported 2 subsequent pregnancies, and 2 (3.3\%) women reported 3 subsequent pregnancies. All pregnancies currently reported in the registry are singleton gestations. Of the women with 
reported subsequent pregnancies, only one reported any additional uterine surgery prior to OMFS. Of the 77 reported pregnancies, 15 are ongoing or missing outcome data, leaving 62 pregnancies for analysis (Figure 1). Spontaneous pregnancy loss prior to 20 weeks occurred in 10 patients (13.0\%) with median gestational age of pregnancy loss of 10.0 weeks (5.911.0); no elective terminations of pregnancy were reported. The only major fetal anomaly noted in the subsequent pregnancies was one recurrent open neural tube defect. In all pregnancies reported in this data, the uterine stapler was used to create the hysterotomy at the time of the fetal surgery.

The outcomes of the 52 pregnancies that progressed beyond 20 weeks EGA are described in table 2. Among the pregnancies progressing beyond 20 weeks, the median EGA at delivery was 37 (36.3-37) weeks. The liveborn rate among pregnancies progressing beyond 20 weeks EGA is $96.2 \%$ (50/52), and is $80.6 \%$ (50/62) for the entire cohort. All pregnancies were delivered by cesarean delivery, $4(7.7 \%)$ by classical hysterotomy and the remainder reported delivery via low segment transverse incision. Gestational diabetes was reported in one pregnancy and no cases of hypertensive disorders of pregnancy were reported.

Uterine rupture occurred in $5(9.6 \%)$ subsequent pregnancies, all in a first pregnancy following OMFS. The median gestational age at time of uterine rupture was 28.0 weeks (26.0-31.5), with uterine ruptures noted at 26, 26, 28, 31, and 32 weeks EGA. Two (40\%) of the uterine rupture cases ( 26 weeks, 31 weeks 6 days) resulted in fetal death (fetal death prevalence $3.8 \%$ ), while a liveborn neonate was delivered in the other 3 cases. Inter-delivery interval, the status of the hysterotomy at the time of delivery of the fetal surgery pregnancy and gestational age of delivery of the fetal surgery pregnancy were not associate with uterine rupture (Table 3). There was no consistent location of the uterine hysterotomy at the time of OMFS in the subsequent pregnancies that were complicated by uterine rupture, with OMFS incision sites described as posterior fundal (2), posterior transverse (2), and anterior fundal (1) incisions. In an additional 9 pregnancies (17.3\%) the prior fetal surgery hysterotomy at the time of subsequent delivery was described as thin or as a dehiscence, but were asymptomatic. Placenta previa was noted in one subsequent pregnancy and placenta accreta was reported in one additional subsequent pregnancy, none requiring hysterectomy. Maternal blood transfusion at delivery was reported in 4 subsequent pregnancies $(7.7 \%)$ and no other maternal complications or maternal deaths were reported in this cohort.

\section{Comment:}

\section{Principal findings:}

The fMMC Consortium subsequent pregnancy outcome data suggests that in most cases, pregnancy following OMFS for fMMC closure results in favorable maternal and fetal outcomes with low rates of preterm birth prior to 32 weeks and a low rate of maternal morbidity with a transfusion rate of $7 \%$ the only reported maternal morbidity. The risk of uterine rupture of $9.6 \%$, with 2 uterine ruptures resulting in fetal death of potentially viable gestations, deserves attention. This data is to date the largest cohort of subsequent pregnancy outcomes among women undergoing OMFS for fMMC. 


\section{Clinical implications:}

Complications of the hysterotomy incision is not an unanticipated problem among those familiar with OMFS and several approaches to reduce the morbidity associated with uterine rupture have been suggested. The ideal duration for optimal healing of the fetal surgery hysterotomy incision is unknown. Data from trial of labor after cesarean delivery studies suggest that inter-delivery intervals less than 18 months are associated with a 3-fold increased risk of uterine rupture (OR 3.0, 95\% CI 1.3,7.2)[10, 11], while intervals between 18 and 24 months or $>24$ months are not associated with increased risk of uterine rupture. This data is predominantly applied in decision making regarding attempted vaginal delivery after prior cesarean and given that the uterine incision in OMFS more approximates that of a classical cesarean hysterotomy, trial of labor in subsequent pregnancy is not recommended. No other data exists to address optimal inter-delivery interval for hysterotomy healing. Thus, based on this prior cesarean data, many fetal programs recommend an inter-delivery interval of $>24$ months in women who desire subsequent pregnancy after OFMS to reduce the risk of uterine incision complications.

Given the known risk of uterine rupture following OFMS, which could be compared to a prior classical cesarean delivery, elective delivery is recommended by most fetal surgery programs at 36-37 weeks to reduce the risk of uterine rupture associated with labor [12, 13] while balancing the risks of iatrogenic prematurity, however the rate of uterine rupture prior to labor requires additional investigation. Prior OMFS is thus considered an indication for late preterm/early term delivery with potential fetal risks outweighing the benefits of delayed delivery to term (39 weeks 0 days)[14]. Finally, given the risk of uterine rupture following OMFS for fMMC closure, a high index of suspicion for uterine incision complications should be considered in such patients presenting with preterm labor symptoms, vaginal bleeding in pregnancy, or uterine/abdominal pain and close, likely inpatient, observation is warranted.

As more experience with fetal surgery for MMC is gained, attention to the initial procedure technique may ameliorate future pregnancy risk. Variable approaches to both surgical technique for fetal surgery, such as minimally invasive procedures or alternative hysterotomy closure technique, may result in reduced rates of uterine incision complications[15]. Fetoscopic closure of MMC has demonstrated feasibility and the smaller incisions on the uterus may allow vaginal delivery, which should also translate to lower maternal risk in subsequent pregnancies [16-18]. As the number of fetoscopic procedures remain limited, long term data on neonatal outcomes such as neurologic function, reversal of hindbrain herniation, CSF leak, and need for reoperation are also more limited than in open fetal surgery. Thus, OMFS remains the most evidence-based approach for fMMC closure given the published level 1 data [1].

In the US 1645 babies are delivered annually with spina bifida [19]. Fetal surgery has demonstrated significant reduction in the lifetime morbidity associated with MMC and thus clearly has established a role as a standard of care management option for these pregnancies [20]. As the availability of fetal surgery for fMMC closure increases, there will be an increasing proportion of women seeking care with subsequent pregnancies. Many of these pregnancies will receive care by providers with less experience in fetal surgery, thus 
understanding the risks associated with these pregnancies is critical. Clear guidelines to the obstetric management of pregnancies subsequent to OMFS should be developed and widely distributed to obstetric providers. Such recommendations for care include 1) collaborative care with a center with expertise in OMFS both in the preconception period and during the subsequent pregnancy is likely beneficial to ensure up to date pregnancy recommendations as more data is obtained, 2) discussion of the recommendations for inter-delivery interval of $>24$ months and availability of contraception as indicated, 3) folic acid supplementation of $4 \mathrm{mg}$ daily prior to conception, 4) prenatal screening for MMC using serum analytes or ultrasound, 5) frequent provider contact during the pregnancy to assess for symptoms of preterm labor or uterine rupture, and 6) planned delivery via cesarean at 36 0/7 - 37 6/7 weeks EGA.

\section{Research implications:}

Given the limited detail of the pregnancy outcome data and small sample size in this cohort, more precise information in determination of risk factors for uterine rupture cannot be defined, nor is there sufficient information to determine if there were clinical signs that could allow intervention to prevent the fetal deaths. Additionally, alternative uterine entry and closure techniques, including hysteroscopic fMMC repair may influence subsequent pregnancy outcomes and uterine rupture risk. Thus, additional research into direct comparison of these techniques, including comparison of neonatal and childhood outcomes based on fMMC repair technique are needed to ensure the fetal repair techniques result in comparable outcomes, as well as maternal outcomes. Centers performing fMMC repair of any type should be encouraged to participate in registries such as the fMMC Consortium registry to contribute to more robust patient population and thus allow such comparisons to be available. Given the significance of this data and areas of detail that require additional exploration, expanded research funding into the outcomes of these pregnancies should be prioritized, including extramural funding to support national and international registries such as the fMMC consortium registry.

\section{Strengths and weaknesses:}

This data does have several strengths and limitations. This registry does not include data on intended fertility following OMFS for fMMC closure thus fertility rates and risk of infertility following open fetal surgery are not able to be estimated in this cohort. Limited data have not suggested an association between OMFS and infertility [4], however this is an area worthy of additional investigation. Additionally, the sample size is insufficient to evaluate the risk of abnormally adherent placenta. Increasing numbers of cesarean deliveries are associated with increased risk of placenta previa/accreta [21]. Given that fetal surgery is associated with two uterine incisions (fetal surgery and delivery) during the affected pregnancy, open fetal surgery potentially poses a similar increased risk of morbidly adherent placenta in subsequent pregnancies. The subsequent pregnancy outcome data is obtained by study coordinator-initiated survey with backup medical record data review as is available. The patient report of this data limits the degree of detail available which may limit the conclusions made from this cohort. Finally, many patients in this registry may have unreported pregnancies due to lost to follow up with the study institutions. Lack of inclusion of these pregnancies can affect the precision of the estimates of the reported outcomes, 
however the data in this cohort is consistent both with prior reported data following OMFS and is consistent with data reported from pregnancy outcomes following classical cesarean deliveries. Finally, the population racial and ethnic diversity in this data set is limited, although similar to the MOMS cohort. This could lead to a potential lack of generalizability and inability to determine ethnic factors associated with pregnancy outcomes, and should prompt investigation into availability of OMFS for diverse populations.

Despite these limitations, this cohort represents the largest group to date of subsequent pregnancy outcomes following OMFS for fMMC closure, providing a reliable estimate of pregnancy outcomes, and thus is critical information to allow families and providers to participate in shared decision making as they consider open fetal surgery for MMC.

\section{Conclusions:}

While most pregnancies subsequent to OMFS result in good maternal and neonatal outcomes with early pregnancy loss rates comparable to the general population [22] and live birth among pregnancies progressing beyond 20 weeks of $96 \%$, the risk of uterine rupture or dehiscence with associated potential for fetal morbidity is significant and is similar to that of prior classical uterine incisions [3]. Preoperative counseling for patients considering maternal-fetal surgery for fMMC closure should include not only the benefits for reduction in MMC morbidity for the neonate and child, but the associated maternal risks for the index pregnancy, as well as the potential additive risk of uterine incision complications in future pregnancies, allowing the family the opportunity to decline fetal surgery. Subsequent pregnancy management with appreciation for these risks, such as delivery at 36-37 weeks gestational age and care in close collaboration with a center with expertise in OMFS, is recommended. Further investigation into fertility rates and abnormal placentation, as well as pregnancy outcomes with alternative uterine entry techniques for fetal surgery is warranted.

\section{Acknowledgements:}

The authors would like to recognize Karen Dorman, Research Coordinator, Maternal Fetal Medicine and Maya Lindley Center for Maternal and Infant Health, University of North Carolina - Chapel Hill for their assistance in the performance of this study.

The North American Fetal Therapy Network and the fMMC Consortium are supported through funding by the Eunice Kennedy Shriver National Institute of Child Health and Human Development (5R131HD059293-05)

\section{References:}

1. Adzick NS, Thom EA, Spong CY, Brock JW, Burrows PK, Johnson MP, Howell LJ, Farrell JA, Dabrowiak ME, Sutton LN, Gupta N,Tulipan NB, D’Alton ME, Farmer DL, MOMS Investigators A randomized trial of prenatal versus postnatal repair of myelomeningocele. N Engl J Med, 2011 364(11): p. 993-1004. [PubMed: 21306277]

2. Landon MA, Lynch CD, Optimal timing and mode of delivery after cesarean with previous classical incision or myomectomy; a review of the data. Seminars in Perinatology, 2011 35: p. 257-261. [PubMed: 21962624]

3. Chauhan SP, Magann EF, Wiggs CD, Barrilleaux PS, Martin JN Jr., Pregnancy after classic cesarean delivery. Obstet Gynecol, 2002 100(5 Pt 1): p. 946-50. [PubMed: 12423858]

4. Wilson RD, Lemerand K, Johnson MP, Flake AW, Bebbington M, Hedrick HL, Adzick NS, Reproductive outcomes in subsequent pregnancies after a pregnancy complicated by open maternalfetal surgery (1996-2007). Am J Obstet Gynecol, 2010 203(3): p. 209.e1-6. [PubMed: 20537307] 
5. Zamora IJ, Ethun CG, Evans LM, Olutoye OO, Ivey RT,Haeri S,Belfort MA,Lee TC,Cass DL, Maternal morbidity and reproductive outcomes related to fetal surgery. J Pediatr Surg, 2013 48(5): p. 951-5. [PubMed: 23701766]

6. Thom EA, Maternal reproductive outcomes after in-utero repair of myelomeningocele. Am J Obstet Gynecol, 2016 214(1 Supp (Abstract 50)): p. 36.

7. Harris PA, Taylor R, Thielke R, Payne J, Gonzalez N, Conde JG, Research electronic data capture (REDCap) - A metadata-driven methodology and workflow process for providing translational research informatics support. J. Biomed Inform, 2009 42(2): p. 377-81. [PubMed: 18929686]

8. Moise KJ, Moldenhauer JS, Bennett KA, Goodnight W, Luks FI, Emery SP, Tsao K, Moon-Grady AJ, Moore RC, Treadwell MC, Vlastos EJ, Wetjen NM, Current selection criteria and perioperative therapy used for fetal myelomeningocele surgery. Obstet Gynecol, 2016 127(3): p. 593-7. [PubMed: 26855109]

9. Landon MB HJ, Leveno KJ, Spong CY, et al. , National Institute of Child Health and Human Development Maternal-Fetal Medicine Units Network. Maternal and perinatal outcomes associated with a trial of labor after prior cesarean delivery. N Engl J Med, 2004 16(351(25)): p. 2581-2589.

10. Shipp TD, Zelop CD, Repke JT, Cohen A, Lieberman E, Interdelivery interval and risk of symptomatic uterine rupture. Obstet Gynecol, 2001 92(2): p. 175.

11. Bujold E, Gauthier RJ, Risk of uterine rupture associated with an interpregnancy interval between 18 and 24 months. Obstet Gynecol, 2010 115(5): p. 1003. [PubMed: 20410775]

12. Spong CY, Mercer BM, D’alton M, Kilpatrick S, Blackwell S, Saade G, Timing of indicated latepreterm and early-term birth. Obstet Gynecol, 2011 118: p. 323. [PubMed: 21775849]

13. Medically indicated late-preterm and early-term deliveries. ACOG Committee Opinion No. 560. American College of Obstetricians and Gynecologists. Obstet Gynecol, 2013 121(908-10).

14. Tita AT, Landon MB, Spong CY, Lai Y, Leveno KJ, Varner MW, et al., Timing of elective repeat cesarean delivery at term and neonatal outcomes. Eunice Kennedy Shriver NICHD Maternal-Fetal Medicine Units Network. N Engl J Med, 2009 360: p. 111-20. [PubMed: 19129525]

15. Zaretsky MV Liechty KW, G. HL, Behrendt NJ, Reeves S, Marwan AI, Wilkinson C,Handler M, Lagueux M, Crombleholme TM,, Modified Hysterotomy Closure Technique for Open Fetal Surgery. Fetal Diagn Ther, 201844.

16. Kohn JR RV, Sellner AA, Sharhan D, Espinoza J, Shamshirsaz AA, Whitehead WE, Belfort MA, Sanz Cortes M, Management of Labor and Delivery After Fetoscopic Repair of an Open Neural Tube Defect. Obstet Gynecol, 2018 131: p. 1062-1068. [PubMed: 29742660]

17. Belfort MA, Whitehead WE, Shamshirsaz AA, Bateni ZH, et al., Fetoscopic Open Neural Tube Defect Repair: Development and Refinement of a Two-Port, Carbon Dioxide Insufflation Technique. Obstet Gynecol, 2017 129(4): p. 734-743. [PubMed: 28277363]

18. Araujo Junior E, Eggink AJ, van den Dobbelsteen J Martins WP, Oepkes D, Procedure-related complications of open vs endoscopic fetal surgery for treatment of spina bifida in an era of intrauterine myelomeningocele repair: systematic review and meta-analysis. Ultrasound Obstet Gynecol, 2016 48(2): p. 151-60. [PubMed: 26612040]

19. Williams J, Mai CT, Mulinare J, Isenburg J, et al. , Updated Estimates of Neural Tube Defects Prevented by Mandatory Folic Acid Fortification — United States, 1995-2011. MMWR Morb Mortal Wkly Rep, 201564.

20. Maternal-fetal surgery for myelomeningocele. ACOG Committee Opinion No. 720. American College of Obstetricians and Gynecologists. Obstet Gynecol, 2017 130: p. 164-7.

21. Silver RM, Landon MB, Rouse DJ, et al., Maternal morbidity associated with multiple repeat cesarean deliveries. Obstet Gynecol, 2006 107: p. 1226. [PubMed: 16738145]

22. Early pregnancy loss. ACOG Practice Bulletin No. 200. American College of Obstetricians and Gynecologists. . Obstet Gynecol, 2018 132:e197-207. [PubMed: 30157093] 


\section{AJOG at a Glance}

\section{A. Why was this study conducted?}

This study describes the maternal and neonatal outcomes in pregnancies subsequent to a pregnancy managed with open maternal fetal surgery (OMFS) for fetal myelomeningocele (fMMC).

\section{B. What are the key findings?}

In pregnancies subsequent to OMFS for MMC, the live birth rate in pregnancies progressing after 20 weeks is $96.2 \%$ with a median gestational age at delivery of 37 weeks. Uterine rupture of the OMFS incision was noted in $9.6 \%$ of subsequent pregnancies and fetal death occurred in 2 of the 5 cases of uterine rupture. The only maternal morbidity was a rate of transfusion of $7.7 \%$

\section{What does this study add to what is already known?}

This data provides patients and providers an understanding of the outcomes of pregnancies after OMFS which should be used during the initial consultation when considering OMFS for fMMC, as well as guidance for management of any subsequent pregnancy. 


\section{Condensation:}

Pregnancies subsequent to open maternal-fetal surgery have an increased risk for complications related to the fetal surgery incision, the most significant being a risk of uterine rupture of $10 \%$ that carries significant risk of fetal morbidity. Counseling prior to fetal surgery should include the potential risk on future pregnancy outcomes. 


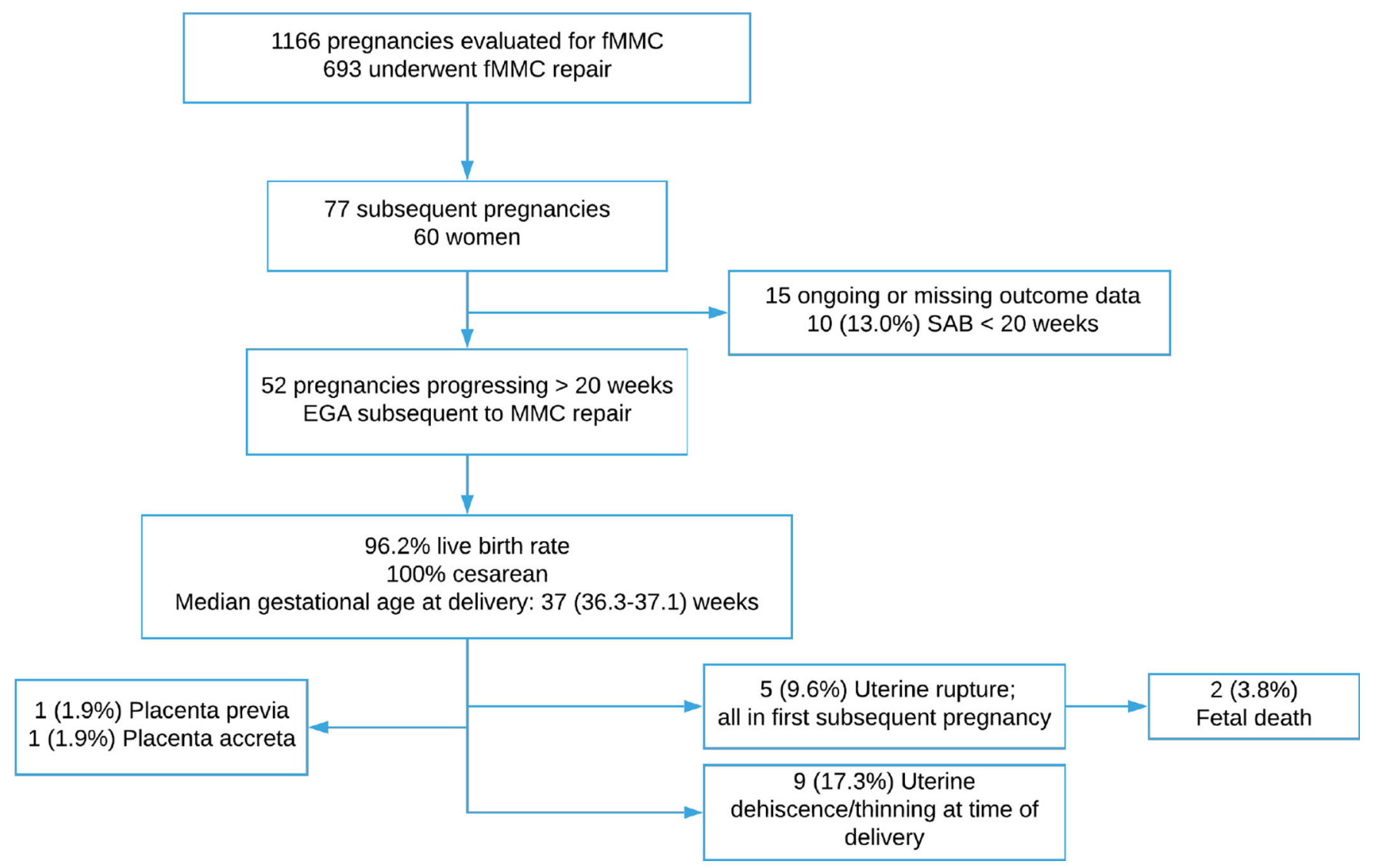

Figure 1:

Pregnancy subsequent to OMFS for fetal MMC 
Table 1:

Demographics of 48 women reporting 62 pregnancies following open maternal fetal surgery for MMC, complete data. N (\%), median (interquartile range); Percent may not $=100 \%$ due to missing outcome.

\begin{tabular}{|c|c|}
\hline Demographics & \\
\hline BMI & $25.6(22.8-27.5)$ \\
\hline Maternal age at fetal surgery pregnancy & $27.5(24.0-30.0)$ \\
\hline Primiparous at fetal surgery pregnancy & $32(66.7)$ \\
\hline \multicolumn{2}{|l|}{ Ethnicity } \\
\hline White & $42(87.5)$ \\
\hline Other & $1(2.1)$ \\
\hline Hispanic & $5(10.4)$ \\
\hline EGA delivery MMC pregnancy (weeks) & $35.4(31.4-37.0)$ \\
\hline \multicolumn{2}{|l|}{ Incision status at MMC delivery } \\
\hline Thin & $12(25.0)$ \\
\hline Dehiscence & $4(8.3)$ \\
\hline Intact & $29(60.4)$ \\
\hline
\end{tabular}




\section{Table 2:}

Subsequent pregnancy outcomes; pregnancies with complete outcome data (52). N (\%), median (IQR). EGA estimated gestational age

\begin{tabular}{lc}
\hline \multicolumn{1}{c}{ Outcome } & \\
\hline Inter-delivery interval (years) $\mathbf{1}^{\text {st }}$ pregnancy (37) & $3.2(2.6-3.7)$ \\
Inter-delivery interval (years) $\mathbf{2}^{\text {nd }}$ pregnancy (5) & $4.6(3.9-5.5)$ \\
Pregnancy loss < 20 weeks & $10(13.0)$ \\
Live birth in pregnancies progressing > 20 weeks EGA & $50(96.1)$ \\
Fetal death pregnancies $>$ 20 weeks EGA & $2(3.8)$ \\
Uterine rupture & $5(9.6)$ \\
Uterine incision dehiscence/thinning at delivery & $9(17.3)$ \\
Placenta previa & $1(19)$ \\
Placenta accreta & $1(19)$ \\
Gestational age at delivery $\mathbf{1}^{\text {st }}$ pregnancy & $36.0(36.1-37.0)$ \\
Preterm birth < 32 weeks EGA $\mathbf{1}^{\text {st }}$ pregnancy & $5(9.6)$ \\
Gestational age at delivery $\mathbf{2}^{\text {nd }}$ pregnancy & $37.3(36.9-37.5)$ \\
Preterm birth < 32 weeks EGA all subsequent pregnancies & $5(9.6)$ \\
Preterm labor & $2(3.8)$ \\
Preterm premature rupture of membranes & $1(19)$ \\
Cesarean delivery & \\
Maternal transfusion at delivery & $45(86.5)$ \\
\hline
\end{tabular}


Table 3:

Characteristics of pregnancies complicated by uterine rupture ( 52 pregnancies progressed beyond 20 weeks EGA)

\begin{tabular}{|c|c|c|c|}
\hline & Rupture (5) & No rupture (47) & $\mathbf{p}$ \\
\hline Maternal age at MMC pregnancy & $30.0(23.5-32.0)$ & $28.0(25-29)$ & 0.47 \\
\hline Parity at MMC pregnancy & & & 0.53 \\
\hline Primiparous & $4(100)$ & $22(62.9)$ & \\
\hline$\geq 1$ one Prior pregnancy & $0(0)$ & $13(36.1)$ & \\
\hline BMI at MMC pregnancy & $23.8(22.6-25.8)$ & $26.5(22.8-29.9)$ & 0.25 \\
\hline Prior uterine surgery & $0(0)$ & $2(5)$ & 0.85 \\
\hline Location of MMC hysterotomy & & & 0.75 \\
\hline Anterior fundal & $1(20)$ & $9(22.5)$ & \\
\hline Posterior fundal & $2(40)$ & $13(32.5)$ & \\
\hline Other & $2(40)$ & $11(27.5)$ & \\
\hline Inter-delivery interval (years) & $3.6(3.0-4.2)$ & $2.9(2.4-3.5)$ & 0.43 \\
\hline MMC incision thin or dehiscence at delivery of fetal surgery pregnancy & $1(20 \%)$ & $10(21.3 \%)$ & 0.52 \\
\hline EGA MMC delivery & $33.0(26.0-36.8)$ & $34.7(31.9-37.0)$ & 0.30 \\
\hline
\end{tabular}

